\title{
MRI Enhancement Rate Value
}

National Cancer Institute

\section{Source}

National Cancer Institute. MRI Enhancement Rate Value. NCI Thesaurus. Code C93598.

The numeric value to indicate an increase in voxel signal over time for an MRI, expressed as signal intensity units per second. 